\title{
Fast Exhaustive Search Algorithm for Discovering Relevant Association Rules
}

\author{
Hend Amraoui*, Faouzi Mhamdi ${ }^{\dagger}$, Mourad Elloumi $^{\ddagger}$ \\ $* \dagger \ddagger$ Laboratory of Technologies of Information and Communication and Electrical Engineering (LaTICE), \\ National Higher School of Engineers of Tunis (ENSIT), University of Tunis, Tunisia \\ ${ }^{*}$ Faculty of Science of Tunis, University of Tunis el Manar, Tunis, Tunisia \\ $\dagger$ Higher Institute of Applied Languages and Computer Science of Beja,University of Jendouba, Tunisia \\ *amraoui.hend@yahoo.fr, † faouzi.mhamdi@ensi.rnu.tn, ${ }^{\ddagger}$ Mourad.Elloumi@gmail.com
}

\begin{abstract}
Association Rules Mining (ARM) is one of the most important tasks of Data mining. The purpose of ARM is to discover relationships having an interest between attributes/patterns stored in very large databases. Nowadays several efficient algorithms have been developed by the researchers for the discovery of relevant Association Rules (ARs). These latter are responsible for decision making in several domains, such as medicine, finance, marketing and many other fields. In this paper, we propose a new algorithm based on exhaustive search to find relevant $A R$ to make the decision and to predict the chance of occurring the Diabetes Mellitus (DM). We develop an algorithm to mine data in less time and less complexity without losing information. Finally, we test our approach using a real database to evaluate the efficiency of our algorithm compared to Apriori algorithm.

Index Terms-Association Rules, Fast Exhaustive Search Algorithm, Support, Confidence, Fitness
\end{abstract}

\section{INTRODUCTION}

The task of ARM was introduced for the first time by Agrawal et al., [1] to discover relationships among attributes in databases. From these association rules, several decisions will be taken. The formal notations of ARM were introduced by Agrawal et al., [1].

Let $I=I_{1}, I_{2}, \ldots, I_{m}$ be a set of $m$ different attributes, $T$ be the transaction that comprises a set of items such that $T \subseteq I, D$ be a database with different transactions $T_{s}$. An association rule is an insinuation in the form of $X \Rightarrow Y$, where $X, Y \subset I$ are sets of items termed itemsets, and $X \cap Y=\emptyset . X$ is named antecedent, $Y$ is called consequent. The rule means $X$ implies $Y$.

ARM goes through two steps: The first one is to identify the frequent items using minimum support threshold $s$ and the second one is to generate the relevant rules from these frequent items using minimum confidence threshold $c$. Since the database is large and users concern about only those frequently purchased items, usually thresholds of support and confidence are predefined by users to drop those rules that are not so interesting or useful. The two thresholds can be specified by the users [2]. Support of an association rule is defined as the percentage/fraction of records that contain $X \cap Y$ to the total number of records in the database [2].

$$
\text { Support }(X Y)=\frac{\text { Support count of } X Y}{\text { Total number of transaction in } D}
$$

DOI reference number: 10.18293/SEKE2019-157
Confidence of an association rule is defined as the percentage/fraction of the number of transactions that contain $X \cap Y$ to the total number of records that contain $X$, where if the percentage exceeds the threshold of confidence, an interesting association rule $X \Rightarrow Y$ can be generated [2].

$$
\text { Confidence }(X \mid Y)=\frac{\operatorname{Support}(X Y)}{\operatorname{Support}(X)}
$$

The generation of all the frequent elements is a time consuming task when the number of items is large [3]. For this purpose we developed an algorithm generating association rules in a direct way by maximizing the support/confidence of the rules skipping the frequent itemset generation step. Our approach is then to treat the problem of ARM as a multi-objective optimization problem where the goal is to find ARs while optimizing a fitness function (described below) i.e., it is a function for evaluating rules [4], it shows if an association rule is a good solution or not.

The remainder of the paper is as follows : Section II introduces some previous work on ARM, section III explains problem and technical solutions, section IV describes the proposed algorithm, the different improvements and its performance, in section V experimental results are presented and finally, the paper is concluded in Section VI.

\section{Literature REVIEW}

A large number of previous studies have been focused on the problem of ARM. Agrawal et al., [5] developed Apriori algorithm. It first generates the set of elements from the previous element sets, then, a pruning step was applied on these candidates. Finally, ARs are deduced from the frequent candidates generated in the first step. Apriori algorithm is easy to execute and very simple but suffers from inefficiencies: scanning the database frequently; generating a large number of candidate sets [6] and demanding huge data storage [7].

Zaki [8] developed Eclat algorithm for discovering the set of frequent attributes. Zaki represented the database with a vertical representation to avoid the repeated traverse of the database. He decomposed the original search space into smaller pieces which can be processed independently in mainmemory, and proposed three new search strategies for enumerating the frequent itemsets such as bottom-up search, top-down search, and hybrid search. 
Han et al., [9] introduced Frequent Pattern Growth (FPGrowth) algorithm. Han et al., used three techniques: The first one was to compress the base as much as possible developing an FP-tree to avoid scanning it several times, the second one was to adopt a pattern fragment growth method to avoid the costly generation of a large number of candidate sets, and finally used divide-and-conquer method to decompose the mining task and reduce the search space.

Uno et al., [10] proposed Linear time Closed item set Miner (LCM) algorithm. They constructed tree-shaped transversal routes composed of only frequent closed item sets. LCM algorithm founded all frequent closed item sets in polynomial time per item set, without storing previously obtained closed item sets in memory.

Yuan [6] proposed an improved Apriori algorithm as a remedial to the previous defects. Yuan used a new database mapping way, pruned more candidate elements and used overlap strategy to count support. The improved Apriori algorithm achieves excellent performance by reducing the time consumed in transaction scanning for the generation of candidate itemsets and by reducing the number of transaction to be scanned.

Sheng et al., [11] proposed a novel method by combining the Apriori algorithm and probabilistic graphical model. Sheng et al. avoided the disadvantage that whenever the frequent items are searched the whole data items have to be scanned cyclically. The effectiveness and feasibility of the proposed method in ARM has been proved.

Liu et al. [12] proposed a fast Apriori algorithm, called ECTPPI-Apriori, for processing large datasets. The algorithm uses a parallel mechanism in the ECPI tissue-like $\mathrm{P}$ system. The time complexity of ECTPPI-Apriori is improved to $O(t)$ compared to other parallel Apriori algorithms.

Fu et al. [13] have developed a parallelization algorithm based on the Hadoop framework and the Map Reduce model. The algorithm proved its efficiency in extracting frequent elements and generating ARs from large transactional databases.

\section{MATERIAL AND METHODS}

\section{A. Data Collection}

For experimental evaluation, a bench mark dataset has been selected as input data https://www.kaggle.com/uciml/pimaindians-diabetes-database. The studied population is PIMA Indian population near Phoenix, Arizona, it is known to be one of the communities with the highest percentage of diabetes in the world. Diabetes is a multifactorial disease where multiple rare genetic, environmental and even behavioral variants influence collectively the expression and the prevalence of traits and diseases [14]. That population has been under continuous study since 1965 by the National Institute of Diabetes and Digestive and Kidney Diseases [15]. The dataset is composed of 768 patients, and two classes. The variables are medical measurements of the patient plus age and pregnancy information. The classes are : $\mathrm{CO}$, indicates True Diabetic Test (268) and C1, indicates False Diabetic Test (500) [16].
Amoung the factors, Diabetes Pedigree Function $(D P F)$ was developed by Smith et al. [15], This function takes into consideration genetic factors inherited from ascendants.

$$
D P F=\frac{\sum_{i} K_{i}\left(88-A D M_{i}\right)+20}{\sum_{j} K_{j}\left(A L C_{j}-14\right)+50}
$$

1) $i$ ranges over all relatives, who had developed diabetes by the subject's examination date;

2) $j$ ranges over all relatives, who had not developed diabetes by the subject's examination date;

3) $K_{x}$ is the percent of genes shared by the relative;

4) $A D M_{i}$ is the age in years of relative, when diabetes was diagnosed;

5) $A L C_{j}$ is the age in years of relative $\mathrm{j}$ at the last nondiabetic examination (prior to the subject's examination date);

6) The constants 88 and 14 represent, the maximum and minimum ages at which relatives of the subjects in this study have developed diabetes;

7) The constants 20 and 50 were chosen such that:

- A subject with no relatives would have a $D P F$ value slightly lower than average.

- The $D P F$ value would decrease relatively slowly as young relatives free of diabetes joined the database.

- The DPF value would increase relatively quickly as known relatives developed diabetes.

Table I illustrates an excerpt of data representing medical measures and observations collected after studying a set of (768) patients. The first column contains an ordering number for the patients. The eight next columns represent measures for medical factor:

- Number of times pregnant $: p$,

- Plasma Glucose Concentration at 2 Hours in an Oral Glucose Tolerance Test :g,

- Diastolic Blood Pressure (mm Hg) $: b$,

- Triceps Skin Fold Thickness $(\mathrm{mm}): s$,

- 2-Hour Serum Insulin $(\mathrm{Uh} / \mathrm{ml}): i$,

- Body Mass Index ((Weight in $\left.\mathrm{kg}) /(\text { Weight in } \mathrm{m})^{2}\right): B$,

- Diabetes Pedigree Function : $d$,

- Age (years) : $a$.

The last column describes the fact whether the corresponding patient is actually sick $(C 1)$ or not $(C 0)$.

TABLE I: Medical Measures

\begin{tabular}{|c|c|c|c|c|c|c|c|c|c|}
\hline & $\mathbf{p}$ & $\mathbf{g}$ & $\mathbf{b}$ & $\mathbf{s}$ & $\mathbf{i}$ & $\mathbf{B}$ & $\mathbf{d}$ & $\mathbf{a}$ & Out \\
\hline 1 & 0 & 100 & 70 & 26 & 50 & 30.8 & 0.597 & 21 & $C 0$ \\
\hline 2 & 0 & 100 & 88 & 60 & 110 & 46.8 & 0.962 & 31 & $C 0$ \\
\hline 3 & 0 & 101 & 62 & 0 & 0 & 21.9 & 0.336 & 25 & $C 0$ \\
\hline$\ldots$ & $\ldots$ & $\ldots$ & $\ldots$ & $\ldots$ & $\ldots$ & $\ldots$ & $\ldots$ & & $\ldots$ \\
\hline 768 & 9 & 184 & 85 & 15 & 0 & 30 & 1.213 & 49 & $C 1$ \\
\hline
\end{tabular}

\section{B. Discretization of Numeric Attributes (Factors)}

In our database, the range of each numeric attribute is very wide. To overcome this problem it was decided to turn numeric attributes into discrete ones. Table II illustrates an excerpt for 
different distinguished ranges for each measured criterion. For example, measures for the criterion Age (last line) may range over $\{21,24\}$ or $\{25,30\}$ (in fact also over $\{31,40\},\{41,55\}$ or $\{56,-\}$ which are not represented in the excerpt).

When an interval ends with - , then it admits no upper bound. An interval suffixed by $*$ is the one to be picked up by default when the measured value does not fit into any interval.

The database in Table I can then be discretized by replacing each measured value for a given criterion by the identifier of the interval it fits into. The result is illustrated in Table III.

TABLE II: Medical Measures Ranges

\begin{tabular}{|c|c|c|c|c|}
\hline $\mathbf{C}$ & $\mathbf{R 1}$ & $\mathbf{R 2}$ & $\ldots$ & $\mathbf{R 6}$ \\
\hline $\mathbf{p}$ & $\{0,2\}$ & $\{3,6\}$ & $\ldots$ & \\
\hline $\mathbf{g}$ & $\{0,89.1\}$ & $\{89.2,107.1\}$ & $\ldots$ & $\{165.2,-\}$ \\
\hline $\mathbf{b}$ & $\{0,0\} *$ & $\{1,76.1\}$ & $\ldots$ & $\{98.2,-\}$ \\
\hline$\ldots$ & $\ldots$ & $\ldots$ & $\ldots$ & $\ldots$ \\
\hline $\mathbf{a}$ & $\{21,24\} *$ & $\{25,30\}$ & $\ldots$ & \\
\hline
\end{tabular}

We note that the factor 10 has been chosen to obtain distinct interval ids. This is true in our example because for all factor, the number of intervals never exceeds 9 . In the general case, this factor can be chosen as the closest power of 10 to the maximum number of intervals for factor to ensure interval's id uniqueness.

Uniqueness is needed here to obtain different items representing different factor ranges. This may be useful when considering sets of such items in the algorithms we present in this paper.

TABLE III: Discretized Medical Measures

\begin{tabular}{|c|c|c|c|c|c|c|c|c|c|}
\hline & $\mathbf{p}$ & $\mathbf{g}$ & $\mathbf{b}$ & $\mathbf{s}$ & $\mathbf{i}$ & $\mathbf{B}$ & $\mathbf{d}$ & $\mathbf{a}$ & Out \\
\hline 1 & 11 & 22 & 32 & 43 & 52 & 63 & 73 & 81 & 91 \\
\hline 2 & 11 & 22 & 33 & 44 & 52 & 65 & 74 & 83 & 91 \\
\hline 3 & 11 & 22 & 32 & 41 & 51 & 61 & 72 & 82 & 91 \\
\hline$\ldots$ & $\ldots$ & $\ldots$ & $\ldots$ & $\ldots$ & $\ldots$ & $\ldots$ & $\ldots$ & $\ldots$ & $\ldots$ \\
\hline 768 & 13 & 26 & 33 & 42 & 51 & 63 & 75 & 84 & 92 \\
\hline
\end{tabular}

\section{Problem Description}

Given the representation in Table III of the patients' database it is interesting to analyze which combination of factor ranges implies the most sickness (or no sickness). We may consider combination taking into account all or only subsets of the factor. To represent a given combination of factor ranges and the associated desired outcome (sickness or not) we are using an Association Rule, defined below.

definition 1: Given $A$ a set of integers and $c$ an integer, we define the corresponding Association Rule (or simply Rule), $\gamma$, denoted by $A \Rightarrow c$. $A$ is called the antecedent of $\gamma$ (and denoted by $\mathcal{A}(\gamma)$ ) and $c$ its consequence (and denoted by $\mathcal{C}(\gamma)$ ). The size of $\gamma$ (denoted by $\mathcal{S}(\gamma)$ ) is the size of its antecedent: $\overline{\mathcal{A}(\gamma)}$.

An association rule $\mathcal{A}(\gamma)$ represents the logical implication: "if items in $A$ are all observed on medical measures for a patient then the outcome for that patient is $c "$. definition 2: Given an association rule $\gamma$, a projection of $\gamma$ is a rule $\gamma^{\prime}$, such that $\mathcal{A}\left(\gamma^{\prime}\right)$ is a non-empty subset of $\mathcal{A}(\gamma)$. When $\gamma^{\prime}$ is a projection of $\gamma$ we also say $\gamma$ is an enrichment of $\gamma^{\prime}$.

We note that the encoded database of Table III can be represented by a sequence (array or list) of association rules. Given definitions 1,2 we can now state the problem we propose to investigate.

problem 1: Given:

- a sequence of association rules $\mathcal{P}=\left[A_{p} \Rightarrow c_{p}\right]_{p \in I}$, with the same size $d$, called population,

- an objective function $f$ mapping each projection of an association rule in $\mathcal{P}$ to a real value in $[0,1]$,

- and an integer $s$ between 1 and $d$.

Compute all the projections of size $s$ of associations rules in $\mathcal{P}$ that maximizes the objective function $f$.

We propose to solve the Problem 1 for all possible sizes of association rules. As objective function we will use the so-called fitness, defined below.

definition 3: Given two strictly positive real values $\alpha$ and $\beta$, and a set $\mathcal{P}$ of association rules, the fitness function (denoted fit) maps each projection $\gamma$ of elements in $\mathcal{P}$ to a positive real value $f i t(\gamma)$ as described below:

$$
f i t(\gamma)=\frac{\alpha \times \operatorname{supp}(\gamma)+\beta \times \operatorname{conf}(\gamma)}{\alpha+\beta}
$$

where:

- the support of $\gamma$ denoted by $\operatorname{supp}(\gamma)$ is $\frac{y(\gamma)}{\overline{\mathcal{P}}}$,

- the confidence of $\gamma$ denoted by $\operatorname{con} f(\gamma)$ is $\frac{y(\gamma)}{x(\gamma)}$,

- $x(\gamma)$ is the number of enrichments of $\gamma$ in $\mathcal{P}$,

- and $y(\gamma)$ is the number of enrichments of $\gamma$ in $\mathcal{P}$, having the same consequence as $\gamma$.

We note that for a given association rule $\gamma, \operatorname{supp}(\gamma)$ measures the probability of occurrence of items in $\mathcal{A}(\gamma)$ together with consequence $\mathcal{C}(\gamma)$ in the population, while $\operatorname{con} f(\gamma)$ measures the probability of occurrence of the consequence $\mathcal{C}(\gamma)$ having all items in $\mathcal{A}(\gamma)$ appearing in the rules of the population.

\section{Fast Exhaustive Search for Association Rule Mining ALgORITHM (FES-ARM)}

To solve Problem 1 we propose the following exhaustive search algorithm. We notice that Algorithm 2 may be called by Algorithm 1 many times for the same rule. This is due to the fact that different rules can have common projections. We note that running Algorithm 2 with a rule that already have been treated does not bring more results. The proposed enhancement consists of using a setRulesDone which will hold all the already analyzed rules, and recursion in Algorithm 2 will only be initiated for rules that are not in RulesDone. There is also a second improvement: It tackes a costly part of the treatment : the computation of the fitness function itself. We propose to keep track for all computed fitnesses for all analyzed rules. This is efficient since fitness is likely to be computed many times for the same rule. 


\section{A. FES-ARM Algorithm}

To solve Problem 1 we propose the following FES-ARM algorithm.

1) Compute all projections of rules in the population and keep only those having maximum fitness.

2) Projections of a given rule $\gamma$, are recursively computed by removing at each recursion step only one item from $\mathcal{A}(\gamma)$.
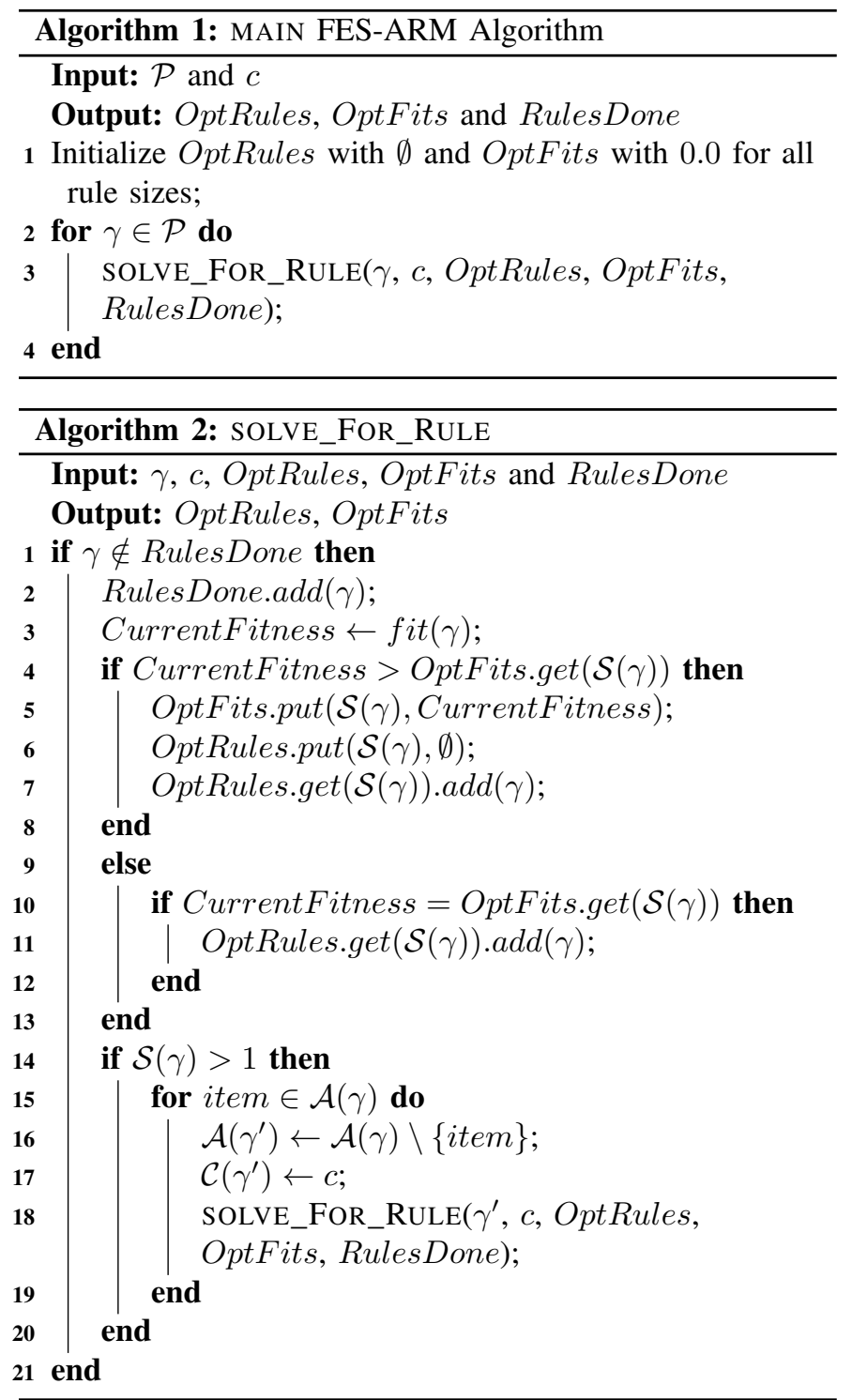

Algorithm 1 takes as input the population $\mathcal{P}$, the considered consequence $c$ and returns a mapping OptRules : $i \rightarrow \Gamma_{i}$ associating to each possible rule size $i$ the set of rules realizing maximum fitness and a mapping OptFits $: i \rightarrow f_{i}$ associating to each possible rule size the actual maximum fitness. It uses a set RulesDone that will contain all analyzed rules (for optimization purpose).

We note that Algorithm 1, solves several instances of Problem 1 at a time, i.e., for all rules sizes.

\section{B. Complexity}

Complexity of Algorithm 1 (using Algorithm 2) will obviously depend on concrete used implementations of mappings and sets data structure.

We propose to use Java HashMap (resp. HashSet) to implement mappings (resp. sets). These classes offer constanttime complexity for regular operations: add, remove and membership test $(\in)$.

This means that all instructions in Algorithm 2 (the recursive call being omitted) are constant-time complexity, except for line 3 where a fitness is computed. Computing the fitness of a rule $\gamma$ means running through the hole population of rules and testing whether $\mathcal{A}(\gamma)$ is a subset of the antecedent of the population member. Testing that a set $A$ is a subset of another set $B$ runs in $O(\min (\bar{A}, \bar{B}))$. This means computing the fitness of $\gamma$ is $O(\mathcal{S}(\gamma) \times \overline{\mathcal{P}})$.

Algorithm 2 will be called for all projections of all rules in the initial population $\mathcal{P}$. There may be redundancy in those generated projections, since two rules can share projections. We note that the if test in line 1 of Algorithm 2 will be executed for all generated projections, with redundancy, while all the other instruction of this algorithm will be executed only once for each distinct generated projection. Each call to Algorithm 2 with rule $\gamma$ and where the if test in line 1 will be positive, will cost $O(\mathcal{S}(\gamma))$.

Let $N_{\Gamma}^{s}$ be the number of all computed projections of size $s$ with redundancy and $N_{\Gamma, d}^{s}$ the number of all the latter projections without redundancy. We can safely infer that complexity of Algorithm 1 is

$$
O\left(\sum_{s \in[1, d]} N_{\Gamma}^{s}+n \times \sum_{s \in[1, d]} N_{\Gamma, d}^{s} \times s\right)
$$

We recall that $d$ is the maximal rule size (in our example it is 8) and we note that $N_{\Gamma, d}^{s}$ and $N_{\Gamma}^{s}$ are likely to be exponential in $d$ and $\overline{\mathcal{P}}$ in the worst case.

\section{Fitness Computation Enhancement}

Computing the fitness for a given rule is a costly task. Indeed we explained in previous sections that it has $O(s \times n)$ complexity, where $s$ is the rule's size and $n$ the population size. We propose to reduce the fitness computation complexity using the following enhancements:

1) Provided that we already have computed a mapping TransactionsByItems

, that sends every item item to the set of the transactions (rules in $\mathcal{P}$ ) $\Gamma($ item $)$ where this item appears. We can compute then compute:

$$
x(\gamma)=\overline{\bigcap_{\text {item } \in \mathcal{A}(\gamma)} \text { TransactionsByItems }(\text { item })}
$$


If we extend the definition of TransactionsByItems to the consequences, we can also compute, for a given sequence $c$ :

$$
x y(\gamma)=\overline{\bigcap_{\text {item } \in \mathcal{A}(\gamma) \cup\{c\}} \text { TransactionsByItems }(\text { item })}
$$

Since the complexity of computing the intersection of two sets $A$ and $B$ runs in $O(\min (\bar{A}, \bar{B}))$. We can infer that the complexity of computing every intersection is $O\left(s \times \operatorname{Min}_{\text {item } \in \mathcal{A}(\gamma) \cup\{c\}}(\right.$ TransactionsByItems $($ item $))$ and that reduces drastically the fitness computation (time) complexity. We note that pre-computing the mapping TransactionsByItems does not introduce too much cost, since it can be done while parsing rules from the database file.

2) We can also keep track of all computed fitnesses so far, using a mapping ComputedFitnesses that sends each already analyzed rule to its fitness value.

\section{EXPERIMENTAL RESULTS}

We propose in this section to discuss results obtained when applying the algorithm presented in this paper compared to Apriori Algorithm. We used databases containing 768, 7680 and 76800 entries, with antecedents of size 8 and 2 consequences, and executed 10 times.

- N: Number of iterations.

- C: The considered consequence.

- A.E.T : Average Execution Time

All times are measured in milliseconds $(m s)$ except when a different unit is explicitly specified.

TABLE IV: Comparative table of Average Execution Time (A.E.T) provided by our Algorithm and Apriori Algorithm

\begin{tabular}{|c|c|c|c|}
\hline $\mathbf{n}$ & $\mathbf{C}$ & A.E.T Apriori & A.E.T FES-ARM \\
\hline \multirow{2}{*}{768} & 91 & 667035 & 2120 \\
\cline { 2 - 2 } & 92 & 852392 & 2766 \\
\hline \multirow{2}{*}{7680} & 91 & & 12855 \\
\cline { 4 - 4 } & 92 & \multirow{3}{*}{$>48 h$} & 13342 \\
\hline \multirow{2}{*}{76800} & 91 & & 145355 \\
& 92 & & 143346 \\
\hline
\end{tabular}

Discussion: In this work we introduce an efficient algorithm to identify relevant ARs. Our experimental evaluation shows that comlexity goes from exponential complexity $O\left(2^{n}\right)$ to polynomial complexity $O\left(\sum_{s \in[1, d]} N_{\Gamma}^{s}+n \times\right.$ $\left.\sum_{s \in[1, d]} N_{\Gamma, d}^{s} \times s\right)$ in comparison with Apriori Algorithm (worst case) and in response time (mentioned in table IV). Compared to Apriori Algorithm, our approach proved the same efficiency in quality of generated rules with maximizing the fitness function, proving that we have not lost the quality of information as well even with the increased size of the database. These results are due to three improvements. The first one consists of using a setRulesDone which will hold all the already analyzed rules, to avoid analyzing the same rule several times (We propose mining specific to general).
The second one is computing the fitness of each transaction by pre-computing the mapping TransactionsByItems while parsing rules from the database file. The third one (It tackes a costly part of the treatment) consists of keeping track for all computed fitnesses for all analyzed rules. This was efficient since fitness was likely to be computed many times for the same rule.

\section{CONCLUSION}

This paper presents a new Fast Exhaustive Search algorithm FES-ARM for discovering efficient ARs to predict the chance of occurring the Diabetes Mellitus (DM). The proposed algorithm produces in a correct way relevant association rules (The same rules produced by Apriori algorithm) while optimizing the average execution time in the worst case to $314,64 \%$. FES-ARM is tested on real databases containing 768,7680 and 76800 entries, with antecedents of size 8 and 2 consequences, and executed 10 times. The complexity of FES-ARM Algorithm is improved from $O\left(2^{n}\right)$ to $O\left(\sum_{s \in[1, d]} N_{\Gamma}^{s}+n \times\right.$ $\left.\sum_{s \in[1, d]} N_{\Gamma, d}^{s} \times s\right)$ in comparison with Apriori Algorithm. Our ambition for the future works, dealing with large transactional databases, is to develop new metaheuristics approaches to solve association rules mining as a combinatorial optimization problem.

\section{REFERENCES}

[1] R. Agrawal, T. Imieliński, and A. Swami, "Mining association rules between sets of items in large databases," in Acm sigmod record, vol. 22, no. 2. ACM, 1993, pp. 207-216.

[2] Q. Zhao and S. S. Bhowmick, "Association rule mining: A survey," Nanyang Technological University, Singapore, 2003.

[3] A. Mukhopadhyay, U. Maulik, S. Bandyopadhyay, and C. A. C. Coello, "Survey of multiobjective evolutionary algorithms for data mining: Part ii," IEEE Transactions on Evolutionary Computation, vol. 18, no. 1, pp. 20-35, 2014.

[4] H. R. Qodmanan, M. Nasiri, and B. Minaei-Bidgoli, "Multi objective association rule mining with genetic algorithm without specifying minimum support and minimum confidence," Expert Systems with applications, vol. 38, no. 1, pp. 288-298, 2011.

[5] R. Agrawal, R. Srikant et al., "Fast algorithms for mining association rules," in Proc. 20th int. conf. very large data bases, VLDB, vol. 1215, 1994, pp. 487-499.

[6] X. Yuan, "An improved apriori algorithm for mining association rules," in AIP Conference Proceedings, vol. 1820, no. 1. AIP Publishing, 2017, p. 080005.

[7] B. Wang, D. Chen, B. Shi, J. Zhang, Y. Duan, J. Chen, and R. Hu, "Comprehensive association rules mining of health examination data with an extended fp-growth method," Mobile Networks and Applications, vol. 22, no. 2, pp. 267-274, 2017.

[8] M. J. Zaki, "Scalable algorithms for association mining," IEEE transactions on knowledge and data engineering, vol. 12, no. 3, pp. 372-390, 2000.

[9] J. Han, J. Pei, and Y. Yin, "Mining frequent patterns without candidate generation," in ACM sigmod record, vol. 29, no. 2. ACM, 2000, pp. $1-12$.

[10] T. Uno, T. Asai, Y. Uchida, and H. Arimura, "Lcm: An efficient algorithm for enumerating frequent closed item sets." in Fimi, vol. 90. Citeseer, 2003.

[11] G. Sheng, H. Hou, X. Jiang, and Y. Chen, "A novel association rule mining method of big data for power transformers state parameters based on probabilistic graph model," IEEE Transactions on Smart Grid, vol. 9 no. 2, pp. 695-702, 2018.

[12] X. Liu, Y. Zhao, and M. Sun, "An improved apriori algorithm based on an evolution-communication tissue-like p system with promoters and inhibitors," Discrete Dynamics in Nature and Society, vol. 2017, 2017. 
[13] C. Fu, X. Wang, L. Zhang, and L. Qiao, "Mining algorithm for association rules in big data based on hadoop," in AIP Conference Proceedings, vol. 1955, no. 1. AIP Publishing, 2018, p. 040035.

[14] H. Amraoui, F. Mhamdi, and M. Elloumi, "Survey of metaheuristics and statistical methods for multifactorial diseases analyses," AIMS Med Sci, vol. 4, pp. 291-331, 2017.

[15] J. W. Smith, J. Everhart, W. Dickson, W. Knowler, and R. Johannes, "Using the adap learning algorithm to forecast the onset of diabetes mellitus," in Proceedings of the Annual Symposium on Computer Application in Medical Care. American Medical Informatics Association, 1988, p. 261.

[16] L. Breiman, "Bagging predictors," Machine learning, vol. 24, no. 2, pp. 123-140, 1996. 